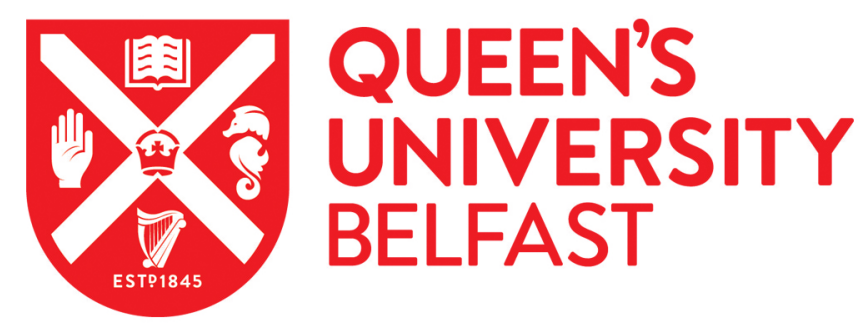

\title{
Ambient mass spectrometry as a tool to determine poultry production system history: a comparison of rapid evaporative ionisation mass spectrometry (REIMS) and direct analysis in real time (DART) ambient mass spectrometry platforms
}

Birse, N., Chevallier, O., Hrbek, V., Kosek, V., Hajŝlová, J., \& Elliott, C. (2021). Ambient mass spectrometry as a tool to determine poultry production system history: a comparison of rapid evaporative ionisation mass spectrometry (REIMS) and direct analysis in real time (DART) ambient mass spectrometry platforms. Food Control, 123, [107740]. https://doi.org/10.1016/j.foodcont.2020.107740

Published in:

Food Control

Document Version:

Peer reviewed version

Queen's University Belfast - Research Portal:

Link to publication record in Queen's University Belfast Research Portal

\section{Publisher rights}

Copyright 2020 Elsevier.

This manuscript is distributed under a Creative Commons Attribution-NonCommercial-NoDerivs License

(https://creativecommons.org/licenses/by-nc-nd/4.0/), which permits distribution and reproduction for non-commercial purposes, provided the author and source are cited

\section{General rights}

Copyright for the publications made accessible via the Queen's University Belfast Research Portal is retained by the author(s) and / or other copyright owners and it is a condition of accessing these publications that users recognise and abide by the legal requirements associated with these rights.

The Research Portal is Queen's institutional repository that provides access to Queen's research output. Every effort has been made to ensure that content in the Research Portal does not infringe any person's rights, or applicable UK laws. If you discover content in the Research Portal that you believe breaches copyright or violates any law, please contact openaccess@qub.ac.uk. 
Ambient mass spectrometry as a tool to determine poultry production system history: a comparison of rapid evaporative ionisation mass spectrometry (REIMS) and direct analysis in real time (DART) ambient mass spectrometry platforms

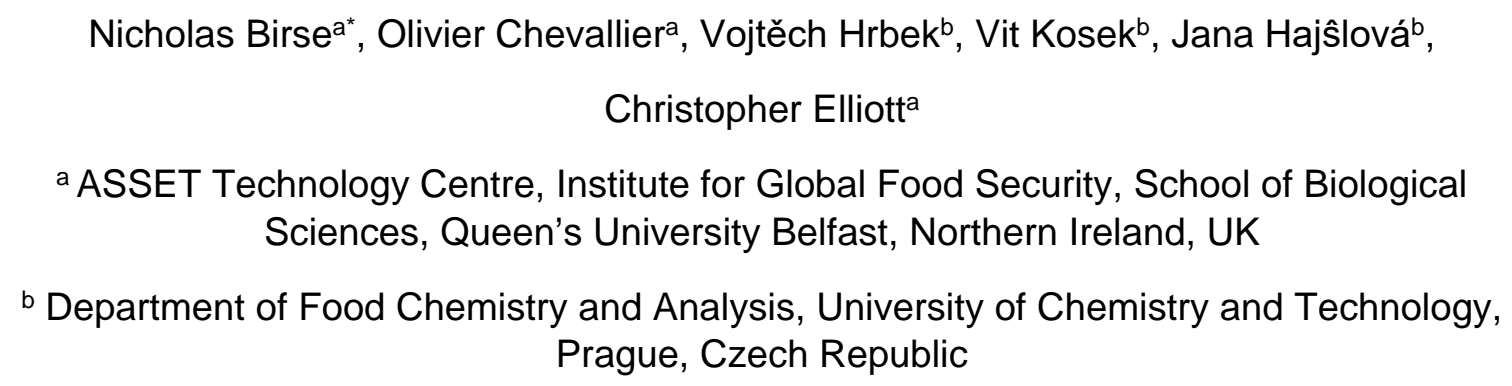

\section{Abstract}

Ambient mass spectrometry are tools of growing interest for the testing of foodstuffs over the fifteen years since the pioneering techniques of desorption electro-spray ionisation (DESI), direct analysis in real time (DART) and atmospheric pressure solid analysis probe (ASAP) were first demonstrated. In this study, rapid evaporative ionisation mass spectrometry (REIMS) was compared with DART to assess whether either system was capable of correctly identifying poultry raised in one of six distinct production systems.

REIMS was able to correctly identify $98 \%$ to $100 \%$ of birds based on their breed. The ability of this platform to identify birds based on their production system ranged from $60 \%$ to $100 \%$, depending on the breed and production system involved. Furthermore, it showed $94 \%$ to $100 \%$ accuracy in identifying premium products and shows substantial promise in detecting mislabelling frauds.

DART was able to identify $100 \%$ of birds based on both breed and production systems, but required extensive sample preparation which impacted throughput, Thus DART is a more suitable technique for laboratory based testing as opposed to factory based. This study has 
shown, for the first time, the capabilities of ambient mass spectrometry as a tool to determine poultry production systems employed.

\section{Keywords}

DART, REIMS, high resolution mass spectrometry, poultry, food authenticity, food fraud, lipidomics

\section{Introduction}

The horse meat scandal which came to light in Europe during 2013 confirmed the worst fears of some industry observers - fraud within the meat supply chain across Europe was widespread and had potentially serious implications for human health.(Annunziata et al., 2018) Investigative journalists have subsequently uncovered further malfeasance within the meat industry, with particular concern being the mislabelling of products with the poultry industry.(Goodley, 2017a, 2017b) The analytical methods used to detect meat and fish frauds, such as DNA analysis, are ineffective at detecting mislabelling of poultry, a result of the breeding and production processes used in poultry production.

Mass spectrometry can detect biomarkers and allergens in food, particularly at the levels needed to ensure consumer safety.(Black, Chevallier, \& Elliott, 2016) It is particularly useful because of the wide range of different compounds it can detect, some simultaneously, from small molecule chemical contaminants through the larger metabolites such as lipids and peptides and proteins.(Kaufmann, 2012) Its use as a testing technique suited to the food industry is limited by the often complex and time consuming sample preparation, extraction and processing techniques which can be required to handle the complex matrices found in many foodstuffs, to concentrate or dilute samples so they're within a range suitable for specific 
instruments, or simply to make the analytes separable and ionisable through techniques such as derivatisation.(Lambropoulou \& Albanis, 2007) (González-Curbelo et al., 2015)

Ambient mass spectrometry systems are an increasingly attractive way for food producers to achieve the benefits of traditional mass spectrometry analysis with the rapid, high throughput needed in an industry where food products can be produced, shipped, purchased and consumed in less time than it would take to perform the aforementioned sample preparation procedures associated with traditional mass spectrometry techniques.(Lu et al., 2018) REIMS and some types of DART analysis can eliminate sample processing entirely, allowing materials taken directly from the production line to be tested within a matter of minutes; other forms of DART analysis, and several other ambient techniques can, whilst not eliminating all sample handling, dramatically reduce the amount of processing necessary to deliver valuable time savings.(Black et al., 2017)(Yong et al., 2017)(Vaclavik, Rosmus, Popping, \& Hajslova, 2010)

REIMS makes use of an electrosurgical diathermy based knife or probe, this device causes the release of cell membrane lipids by joule heating mediated cell lysis, ionisation occurs when the cell membrane material is passed over a heated Kanthal coil and then into the mass spectrometer.(Balog et al., 2015) DART, in contrast, uses a plasma stream to ionise analytes within the sample directly, also formed are charged species in the area surrounding the source which cause secondary ionisation of the analytes.(Cody, Laramée, \& Durst, 2005)

Mass spectrometry, whether used with a chromatography system such as gas chromatography (GC-MS) or liquid chromatography (LC-MS), or directly with an ambient ionisation source, can be operated in two distinct ways; the first is to use the system to detect specific analytes which, in the case of food testing, can be indicative of contamination, fraudulent activity, safety failings or quality concerns.(Lacina, Urbanova, Poustka, \& Hajslova, 
2010) This approach, known as targeted analysis, may additionally include the use of reference standards against which the quantity of the analyte of interest can be measured, a process known as quantitative analysis.(Cajka \& Fiehn, 2016)

The alternative approach is to adopt an untargeted analysis approach.(Riedl, Esslinger, \& Fauhl-Hassek, 2015) This is where a given mass range is scanned, with the acquired spectral data being used to build up a 'fingerprint' of the item being tested.(Araújo et al., 2005)(Møller, Catharino, \& Eberlin, 2005) The acquired spectral data can be generated in both positive and negative polarity modes, differing fractions or parts of a sample may be included and different separation or sample preparation techniques may be undertaken to help build as complete a picture of the chemical composition of the product being tested as is possible.(Yang, Deng, \& Yao, 2015)(Wei, Yao, Ji, Wei, \& Peng, 2013) Chemometric analysis is frequently used to process the resulting untargeted spectral data; this approach allows hundreds or thousands of data files to be analysed and underlying trends within the data to potentially be identified.(Huang et al., 2007) (Gredell et al., 2019) These underlying trends may be obvious and can show specific analytes of interest to pursue for further quantitative analysis using a targeted approach, or they can be discreet, with a number of different analytes all showing very small changes in signal intensity but which when combined form a fingerprint for a given characteristic or trait, enabling the identification of a product which may be adulterated, counterfeit or which has quality problems.(Black et al., 2019) (Raji \& Schug, 2009) (Guitton et al., 2017)

For the present study, two alternative high-resolution ambient mass spectrometry techniques were assessed using poultry breast meat taken from a total of six production systems. REIMS, which required no sample preparation and offered identification of a sample within a matter of a few seconds was compared with DART, which offered significant time savings in the analysis 
of samples, but still required extensive sample preparation prior to analysis. The resulting spectral data was then extracted and processed using the same chemometric modelling package, ensuring consistency of data handling for both techniques.

The use of REIMS to detect food quality, food safety and food fraud incidences has been widely reported previously, with studies using REIMS mainly to identify substitution and adulteration issues.(Black et al., 2019) (Balog et al., 2016) REIMS has also been used previously to report on differences in production systems and to identify quality issues in foodstuffs.(Black et al., 2017) (Verplanken et al., 2017) DART has previously been shown to have significant potential in identifying important differences in poultry feed, and more generally, as a technique which can identify safety, fraud and wider quality issues in foodstuffs.(Cajka, Danhelova, Zachariasova, Riddellova, \& Hajslova, 2013) (Nei, Nakamura, Ishihara, Kimura, \& Satomi, 2017) (Cajka, Riddellova, Tomaniova, \& Hajslova, 2011)

The previous applications of REIMS and DART, combined with the testing requirements for complex production systems led to the design of a study to directly compare the two techniques and determine their respective advantages and disadvantages.

\section{Materials and methods}

\subsection{Chemicals and reagents}

2-propanol (LC-MS grade), methanol (LC-MS grade) and tert-Butyl methyl ether were supplied by Honeywell Riedel-de Haën (Seelze, Germany). Leucine-Enkephalin was obtained from Waters (Millford, MA, USA) and ultra-pure deionised water $(18.2 \mathrm{M} \Omega / \mathrm{cm})$ was obtained from a Millipore Milli-Q system (Billerica, MA, USA).

\subsection{Sample collection and preparation}


199 samples of chicken breast meat were collected directly from a poultry producer, then were refrigerated at $+4^{\circ} \mathrm{C}$ from the point they left the production facility until they were stored at $80^{\circ} \mathrm{C}$. The samples were sourced from six unique production systems; standard plus $(n=38)$, higher welfare $(n=33)$, omega-3 $(n=17)$, organic $(n=38)$, free range $(n=37)$ and free range corn fed $(n=36)$.

The samples were cut into four sections and assigned to one of four groups (A-D) at random immediately prior to being stored at $-80^{\circ} \mathrm{C}$.

The samples for REIMS analysis were then defrosted for approximately 4 hours prior to analysis and then kept at $+4^{\circ} \mathrm{C}$ immediately prior to analysis.

The samples for direct analysis in real time-standard volume and pressure (DART-SVP) analysis were freeze-dried using a Lablyo freeze drier (Frozen in Time, York, UK) for a minimum of 48 hours and then homogenized using a $\mathrm{CH} 180 \mathrm{~A}$ food processor (Kenwood, Havant, Hampshire, UK) to a fine powder. 50mg of the sample powder was weighed into a 1.5mL Eppendorf tube using a Discovery DV215CD balance (Ohaus Europe GmbH, Nanikon, Switzerland).

The analytes were extracted with $1 \mathrm{~mL}$ of ice-cold tert-Butyl methyl ether/methanol $(3: 1, \mathrm{v} / \mathrm{v})$ and vortexed for 30 seconds using a Vortex Genie 2 (Scientific Industries, Bohemia, NY, USA), they were next sonicated for 5 minutes using a camSonix C1275 sonicator (Camlab, Cambridge, UK) at maximum frequency. $450 \mu \mathrm{L}$ Milli-Q water was then added and the sample vortexed for a further 30 seconds. The samples were then stored at $-20^{\circ} \mathrm{C}$ for 10 minutes, finally they were centrifuged at $14,000 \mathrm{~g}$ for 15 minutes at $+4^{\circ} \mathrm{C}$ using a $5415 \mathrm{R}$ bench-top centrifuge (Eppendorf, Hamburg, Germany). The two fractions (polar and non-polar) were then collected in fresh $1.5 \mathrm{~mL}$ Eppendorf tubes and stored at $-20^{\circ} \mathrm{C}$ until ready for analysis.

\subsection{Mass spectrometry configuration}

Two different HRMS systems were employed for this study. The first, a Waters G2-XS QToF (Waters, Wilmslow, UK) was used for REIMS analysis and was fitted with Waters REIMS ion 
source. The second, a Thermo Exactive Orbitrap (Thermo Fisher Scientific, Bremen,

158 Germany) was used for DART-SVP analysis, and was fitted with the Vapur interface and DART-SVP 200 ion-source (IonSense, Saugas, MA, USA).

The REIMS system was operated with the following parameters: negative ionisation mode, cone voltage of 40 volts, heater bias of 40 volts. Leucine Enkephalin $(0.1 \mathrm{ng} / \mu \mathrm{L})$ in 2 -propanol was infused into the REIMS source by means of a Waters Acquity I-Class BSM (Waters, Milford, MA, USA) at a flow rate of $0.2 \mathrm{~mL} /$ minute to enable accurate mass correction to be applied, the presence of 2-propanol additionally assists in ionisation. Data was acquired in negative ionisation mode, in sensitivity mode with continuum data acquisition, over a mass range of $50-1,200 \mathrm{~m} / \mathrm{z}$ with a scan speed 2 scans per second.

The sampling probe used was supplied by Waters (Wilmslow, UK) and consists of a fixed geometry bi-polar probe enabling consistent sampling depth. The probe is connected to the instrument by means of food grade Tygon S3-B44-3 0.5mm ID tubing (Saint-Gobain, Solon, $\mathrm{OH}, \mathrm{USA}$ ) and powered by an Erbe VIO50C electrosurgical generator (Erbe Elektromedizin $\mathrm{GmbH}$, Tuebingen, Germany) set to 30 watts in dry-cut mode. Samples with approximate dimensions of $75 \mathrm{~mm} \times 50 \mathrm{~mm} \times 50 \mathrm{~mm}$ were 'burned' four times using the bi-polar probe, each burn lasted approximately 1 second, with each burn evenly separated from the last.

The mass spectrometer was operated with the following parameters: positive and negative ionisation modes, capillary voltage of \pm 60 volts, tube lens voltage of \pm 120 volts and capillary temperature of $250^{\circ} \mathrm{C}$. The DART-SVP source was operated with the following parameters: positive and negative ionisation modes, grid electrode of \pm 350 volts, discharge needle voltage of -5000 volts, gas temperature of $350^{\circ} \mathrm{C}$. Helium gas was used in running mode with nitrogen being used in standby mode. Data was acquired over a mass range of $50-1,000 \mathrm{~m} / \mathrm{z}$ with a scan speed of 1.5 scans per second.

The sampling mechanism used for the DART-SVP consisted of the $X-Z$ transmission grid module (IonSense, Saugus, MA, USA) which offered 96 discrete sample positions on a 
stainless steel grid, in an 8 row by 12 column format. $3 \mu \mathrm{L}$ of either the polar or non-polar 184 fraction was spotted onto one of the 96 sample positions. The grid was then passed orthogonally through the DART plasma source by means of a two-axis linear rail system; the speed for this was set to $0.5 \mathrm{~mm} / \mathrm{second}$. This process was repeated until all 240 samples had their polar and non-polar fractions analysed in both positive and negative ionisation modes.

\subsection{Data processing}

REIMS data was acquired using MassLynx v4.1 (SCN 949) (Waters, Wilmslow, UK) and imported into Waters AMX (1.0.1563.0) (Waters Research Centre, Budapest, Hungary) where the data was lockmass corrected using the Leucine Enkephalin $\mathrm{m} / \mathrm{z}$ (554.2615). The four burns per sample were averaged, and then using the standard MassLynx pre-processing algorithms, background subtracted and total ion current (TIC) normalised. AMX was used to generate a matrix of normalised TIC values, for this, a TIC threshold of $1 \mathrm{e} 7$ was used. Mass binning was performed at this stage, with a mass bin size of $0.5 \mathrm{Da}$ being used, resulting in a total of 2,300 mass bins.

The resulting matrix file was exported in CSV format and processed further in SIMCA 15.0.2 (Sartorius Stedim Biotech, Umea, Sweden).

DART data was acquired using Excalibur 3.0 (v3.0.63) (Thermo Fisher Scientific, Bremen, Germany) and exported manually using Excalibur's Qual Browser feature to generate a background subtracted, TIC normalised spectrum list for each sample. This data was saved to a CSV file, it was then mass binned (0.5Da, total 1,900 mass bins) and combined by means of a custom R script (VSCHT, Prague, Czech Republic) to generate a matrix file of normalised TIC values directly comparable to those generated using REIMS and capable of being processed in an identical manner using SIMCA 15.0.2.

REIMS and DART-SVP data was imported into SIMCA 15.0.2 where it was mean-centred and grouped into one of six classes, each class representing a distinct production system. REIMS and DART-SVP data was UV scaled. Principal Component Analysis (PCA) modelling, an 
unsupervised modelling technique, was initially performed on the datasets to understand any inherent separation present within the data without explicitly separating samples based on their class assignment. Orthogonal Partial Least Square-Discriminant Analysis (OPLS-DA) supervised models were then generated to observe whether separation could be improved further by discriminating based on class assignment. REIMS models were generated using the $600-900 \mathrm{~m} / \mathrm{z}$ mass range, whilst DART models were generated using the $50-500 \mathrm{~m} / \mathrm{z}$ mass range.

Hierarchical binary modelling was utilised to further improve the predictive ability of the models, here, the REIMS and DART-SVP data was again imported into SIMCA 15.0.2 as before but was assigned to one of two classes, allowing one production system to be analysed against one or more of the other production systems.

DART-SVP datasets were additionally combined and a further series of models generated which make use of both ionisation polarities and both extract fractions.

The validity of the models and their predictive ability was assessed by using $\mathrm{R}^{2}, \mathrm{Q}^{2}$ and Root Mean Squared Error of Cross Validation (RMSECV) values. $\mathrm{R}^{2}$ describes the variance within the model as described by the total number of components present whilst $\mathrm{Q}^{2}$ describes the overall predictive ability of the model independently of the data used to train the model. Permutation testing (200 permutations) was also carried out to ensure that the OPLS-DA models were not over-fitted for the training dataset.

\section{Results}

The results show that both REIMS and DART can both identify individual breeds of bird with cross-validation levels at or near $100 \%$. The two systems show very different levels of ability to distinguish between production systems within the same breed; REIMS cross-validation performance varies from just over $60 \%$ to just under $100 \%$ depending on which production 
systems are involved, a marked contrast to DART which achieves cross-validation performance of $100 \%$ for all production systems.

\subsection{REIMS results}

237 Figure 1 shows the PCA, OPLS-DA and permutations tests for the six class model containing 238 all production systems. It can be seen that that three main groups have formed in both PCA and OPLS-DA plots. One of these groups consists of a single class; the omega-3 birds, one group consists of free range, free range corn fed and organic birds, with the final group consisting of standard plus and higher welfare birds.

Table 1 shows the correct classification rate for this model, which overall is $65.33 \%$. This is lower than expected, the correct classification rate for this model, when taking breed into account was calculated to be $98.99 \%$. The $\mathrm{R}^{2}$ value for this model was 0.496 and the $\mathrm{Q}^{2}$ value 0.455 . The $R^{2}$ value is a measure of the quality of data fit, and the $Q^{2}$ value a measure of the predictive power of the model, values closer to the maximum of 1 indicate increasing quality of data fit or increasing predictive ability.

The high level of accuracy in determining breed suggested that a hierarchical approach to production system identification could be viable, with the first stage of analysis being to identify the breed of bird. To enable this, the three production systems using Ross 308 birds were combined into a single class, with the three production systems using Hubbard birds also combined into a single class. This generated a binary model capable of identifying bird breed, the correct classification rate for this model was $100 \%$ (table S1), with an $\mathrm{R}^{2}$ value of 0.913 and $a Q^{2}$ value of 0.896

The second stage in the hierarchical approach was to build models for either Ross 308 or Hubbard birds, each containing three classes.

The Ross 308 model, comprising the standard plus, higher welfare and omega-3 birds showed excellent ability to correctly identify the omega-3 birds, with clear separation in both PCA (figure $2 \mathrm{a}$ ) and OPLS-DA (figure $2 \mathrm{~b}$ ) models. The cross validation correct classification rate 
for this model was $62.50 \%$, with standard plus and omega-3 birds both having a $100 \%$ correct classification rate (table S2), however all higher welfare birds were misidentified as standard plus birds. The PCA and OPLS-DA models both show standard plus and higher welfare birds having no distinguishable separation. Only the omega-3 birds show good separation in this model. This performance is reflected in the low $\mathrm{R}^{2}$ and $\mathrm{Q}^{2}$ values of 0.422 and 0.408 , and in the permutations test (figure $2 \mathrm{c}$ ).

A second model was generated for Ross 308 birds containing just two classes, standard plus and higher welfare birds. Cross validation of this model gives a correct classification rate of $64.79 \%$, with a $68.42 \%$ correct classification rate for standard plus and $60.60 \%$ for higher welfare (table S3). The R2 and Q2 values for this model were particularly low; 0.197 and 0.098 .

The Hubbard model, comprising organic, free range and free range corn fed birds showed no sign of discrimination in the PCA plot (figure 2d) but did show clear separation in the OPLSDA plot (figure 2e). The R2 value and Q2 values for this model were 0.824 and 0.602 respectively, with permutations test (figure $2 \mathrm{f}$ ) also suggest that the model is not over fitted and that its predictive ability is being correctly assessed. The correct classification rate for this model was $97.3 \%$ (table $2 \mathrm{~b}$ ). The individual correct classification rates were $97.37 \%$ for organic, $94.59 \%$ for free range, and $100 \%$ for free range corn fed (table S4).

\subsection{DART results}

DART analysis differed from REIMS analysis in that a total of five datasets were produced and modelled, four were generated by analysis of the polar and non-polar fractions from the extraction in positive and negative ionisation modes, and the fifth dataset was produced by concatenating those four individual datasets.

The PCA plots for the six group models of all four individual datasets show varying levels of separation (Supplementary Information S1). The corresponding OPLS-DA six group models 
for the four individual datasets show different levels of separation for each of the six different

287 production systems (Supplementary Information S2). It can observed that some models demonstrate superior separation of the Ross 308 based production systems, whilst other models show superior separation of the Hubbard based production systems, additionally whilst omega-3 birds show clear separation on all models, that level of separation differs markedly between models. The $\mathrm{R}^{2}$ values for these models varies from 0.578 to 0.723 , whilst the $\mathrm{Q}^{2}$ value varies from 0.491 to 0.508 .

The four different models, when taken together, demonstrate the potential to separate all six different production systems. In light of this, it was decided to generate a model which was produced by combining the underlying matrices used in the generation of the four individual models. This was undertaken by concatenating the four datasets into one single dataset and using this to generate both PCA and OPLS-DA models containing all six production systems (figures $3 a$ and $3 b$ ).

Table 2 shows the cross validation correct classification performance of the OPLS-DA model as $100 \%$ for correctly predicting breed, and $100 \%$ for predicting production system, with an $\mathrm{R}^{2}$ value of 0.891 and a $Q^{2}$ value of 0.717

The hierarchical approach was repeated to enable a direct comparison of this approach between REIMS and DART systems.

The Ross 308 model, comprising the standard plus, higher welfare and omega-3 birds demonstrates clear separation in both PCA (figure 4a) and OPLS-DA (figure 4b) models. The cross validation correct classification rate for this model was $100 \%$ (table S5) with $\mathrm{R}^{2}$ and $\mathrm{Q}^{2}$ values of 0.952 and 0.797 respectively. The PCA and OPLS-DA models both show standard plus and higher welfare birds having a significant level of separation, with the permutations test (figure 4c) suggesting that the model is valid and not over fitted.

The Hubbard model, comprising organic, free range and free range corn fed birds showed good separation in the PCA plot (figure 4d) and clear separation in the OPLS-DA plot (figure 
4e). The permutations test (figure $4 \mathrm{f}$ ) suggests that the model is not over fitted and that its predictive ability is being correctly assessed. The correct classification rate for this model was $100 \%$ (table S6) with $\mathrm{R}^{2}$ and $\mathrm{Q}^{2}$ values of 0.947 and 0.743 respectively.

\section{Discussion}

The results suggest that REIMS is capable of rapidly and accurately determining several aspects of poultry production, particularly the breed of the birds being used. In addition, the ability of REIMS to accurately identify several of the more profitable, premium production systems (omega-3 enriched, free range corn fed and organic) is encouraging and when combined with the ability to correctly identify bird breed, can be used to detect the most plausible fraud scenario, i.e. that of an inexpensive bird being passed off as a premium product. The limitations of REIMS in identifying all production systems are not a major limitation in this application, but this could be important in other potential applications where the differences in production system are more subtle and the resulting lipid profiles less dissimilar.

DART, like REIMS, demonstrated that it can differentiate different poultry meat production systems based on lipid profiles, also a high level of accuracy. The technique benefits from being able to ionise equally well in positive and negative ionisation modes but suffers from some difficulties with regards to ionisation performance on higher molecular weight lipids. The spectral profile for each sample showed significant differences in what molecular mass range the two techniques were able to detect. DART showed little evidence of ionisation at molecular weights higher than $500 \mathrm{~m} / \mathrm{z}$, in contrast to REIMS which showed good ionisation from $100 \mathrm{~m} / \mathrm{z}$ through to $1250 \mathrm{~m} / \mathrm{z}$. The optimum mass range for each instrument was determined to be 600 $900 \mathrm{~m} / \mathrm{z}$ for REIMS and $50-500 \mathrm{~m} / \mathrm{z}$ for DART, whilst it was possible to model REIMS data from $50-500 \mathrm{~m} / \mathrm{z}$, there were fewer ions detected within that range compared to DART and the 
resulting chemometric models were considerably less accurate, potentially a result of the

338 harsher REIMS source fragmenting lower molecular weight lipids.

339 The most dominant ions in the DART spectral data were largely consistent with those seen by

340 Cajka et al (2013) in their analysis of poultry products, albeit with fewer ions above $500 \mathrm{~m} / \mathrm{z}$ in

341 the non-polar negative ionisation data, likely down to differences in extraction conditions.

342 There was evidence of pyrolysis occurring when attempting to increase the energy of the

343 DART plasma stream through raising the helium temperature towards $450-500^{\circ} \mathrm{C}$, this 344 resulted in some loss of data at the lower end of the mass range and no meaningful increase in data beyond the $500 \mathrm{~m} / \mathrm{z}$ limit previously encountered. This suggests a limit in the plasma stream is reached, beyond which pyrolysis rather than further ionisation occurs.

The differences in performance of each system also need to be weighed against their operational requirements and likely usage.

REIMS allowed direct sampling from chicken breast taken directly from the production line; sample processing wasn't necessary. This would make REIMS a viable option for on-site testing, allowing producers to rapidly establish if mislabelling issues may have occurred and in light of the performance with identifying premium products, can provide assurances that organic, free range or omega-3 products are correctly labelled.

DART includes some of the lengthy sample preparation techniques associated with traditional lipidomic techniques such as GC-MS, the time and equipment demands make it better suited to a routine testing laboratory environment, where it can complement or potentially replace GC-MS for routine analysis of samples.

DART when using the transmission grid system, which has an acquisition time of 2 seconds per sample. The extraction process also enables the rapid analysis of the same sample fractions in different ionisation modes and polarities. 
361 This system can be combined with robotic liquid handling systems to automatically prepare a

36296 sample transmission grid, which in turn can typically then be acquired semi-automatically

363 in around 40 minutes, giving an average sample acquisition time of approximately 25 seconds. This compares favourably to existing lipidomic analysis techniques, such as GC-MS, where individual samples may need chromatographic separations in excess of 1 hour per sample.

\section{Conclusion}

REIMS and DART both offer different approaches to the use of ambient ionization sources in food analysis. REIMS offers a faster but less accurate technique free of sample preparation, making it ideal for deployment within or close to food production and importation facilities, whilst DART offers a faster, highly accurate technique which may begin to rival traditional 'gold-standard' hyphenated techniques such as GC-MS and GC-FID for lipid analysis now some of the challenges around quantification with DART-MS are slowly starting to be overcome. This would leave the technique best suited to a laboratory environment.

The two techniques can be considered, to a great extent, complimentary rather than competitors at this stage. This may change in future; development work on REIMS to improve performance in positive polarity mode, especially if accompanied by rapid polarity switching during data acquisition, could conceivably increase performance significantly. This is more likely in cases where the key differences between samples comes from species which don't ionise in negative ionisation mode, or which better ionise in positive ionisation mode.

DART development may see reductions in processing time by shortening or eliminating the need for extensive sample preparation. The ionisation technique also benefits from compatibility with instruments from a number of competing manufacturers and architectures; it is possible to fit a DART ion source to a triple-quadrupole, a time-of-flight or an Orbitrap instrument. Recently, an interface has been developed to connect the system to a compact and relatively robust single-quadrupole system, the Waters QDa. This has enabled the 
development of the DART-QDa platform as a portable system capable of on-site analysis, however further work is needed to reduce or simplify sample preparation before such portability could be extended to meat analysis, however combining SPME with a portable DART-QDa platform has recently been investigated for food-analysis.(Gómez-Ríos, Vasiljevic, Gionfriddo, Yu, \& Pawliszyn, 2017)

It is also worthy of investigation as to whether the direct sampling diathermy probe used in REIMS analysis can be coupled to the DART ionisation source in some manner, in the hope that this combination would enable the REIMS benefits of rapid sampling without sample preparation to be combined with the assumed less harsh ionisation conditions of the DART source to provide a rapid and extremely accurate food fraud detection system.

\section{Acknowledgements}

This project was funded by the Northern Ireland Department for the Economy and Waters Corporation. This project was also supported by the European Union's Horizon 2020 research and innovation program under grant agreement No 692195 (MultiCoop). We would additionally wish to thank Moy Park for supplying the poultry samples used in this project.

\section{References}

Annunziata, L., Visciano, P., Stramenga, A., Colagrande, M. N., Campana, G., Scortichini, G., ... Compagnone, D. (2018). Investigation of phenylbutazone and its metabolite oxyphenbutazone in horse meat products during years 2013-2017. Drug Testing and Analysis, 10(8), 1251-1257. https://doi.org/10.1002/dta.2386

Araújo, A. S., da Rocha, L. L., Tomazela, D. M., Sawaya, A. C. H. F., Almeida, R. R., Catharino, R. R., \& Eberlin, M. N. (2005). Electrospray ionization mass spectrometry fingerprinting of beer. Analyst, 130(6), 884-889. https://doi.org/10.1039/B415252B 
Balog, J., Kumar, S., Alexander, J., Golf, O., Huang, J., Wiggins, T., ... Takats, Z. (2015). In Vivo Endoscopic Tissue Identification by Rapid Evaporative Ionization Mass Spectrometry (REIMS). Angewandte Chemie, (38), 11211. https://doi.org/10.1002/ange.201502770

Balog, J., Perenyi, D., Guallar-Hoyas, C., Egri, A., Pringle, S. D., Stead, S., ... Takats, Z. (2016). Identification of the Species of Origin for Meat Products by Rapid Evaporative Ionization Mass Spectrometry. Journal of Agricultural and Food Chemistry, 64(23), 4793-4800. https://doi.org/10.1021/acs.jafc.6b01041

Black, C., Chevallier, O., Haughey, S., Balog, J., Stead, S., Pringle, S., ... Elliott, C. (2017). A real time metabolomic profiling approach to detecting fish fraud using rapid evaporative ionisation mass spectrometry. Metabolomics, 13(12), 1. Retrieved from http://queens.ezp1.qub.ac.uk/login?url=http://search.ebscohost.com/login.aspx?direct=t rue $\& d b=e d b \& A N=126653069 \&$ site=eds-live $\&$ scope $=$ site

Black, C., Chevallier, O. P., Cooper, K. M., Haughey, S. A., Balog, J., Takats, Z., ... Cavin, C. (2019). Rapid detection and specific identification of offals within minced beef samples utilising ambient mass spectrometry. Scientific Reports, 9(1), 6295. https://doi.org/10.1038/s41598-019-42796-5

Black, C., Chevallier, O. P., \& Elliott, C. T. (2016). The current and potential applications of Ambient Mass Spectrometry in detecting food fraud. TrAC Trends in Analytical Chemistry, Vol. 82, pp. 268-278. https://doi.org///doi.org/10.1016/j.trac.2016.06.005

Cajka, T., Danhelova, H., Zachariasova, M., Riddellova, K., \& Hajslova, J. (2013). Application of direct analysis in real time ionization-mass spectrometry (DART-MS) in chicken meat metabolomics aiming at the retrospective control of feed fraud. Metabolomics, 9(3), 545-557. https://doi.org/10.1007/s11306-013-0495-Z

Cajka, T., \& Fiehn, O. (2016). Toward Merging Untargeted and Targeted Methods in Mass Spectrometry-Based Metabolomics and Lipidomics. Analytical Chemistry, 88(1), 524- 
Cajka, T., Riddellova, K., Tomaniova, M., \& Hajslova, J. (2011). Ambient mass spectrometry employing a DART ion source for metabolomic fingerprinting/profiling: a powerful tool for beer origin recognition. Metabolomics, 7(4), 500-508. https://doi.org/10.1007/s11306-010-0266-z

Cody, R. B., Laramée, J. A., \& Durst, H. D. (2005). Versatile New Ion Source for the Analysis of Materials in Open Air under Ambient Conditions. Analytical Chemistry, 77(8), 22972302. https://doi.org/10.1021/ac050162j

Gómez-Ríos, G. A., Vasiljevic, T., Gionfriddo, E., Yu, M., \& Pawliszyn, J. (2017). Towards on-site analysis of complex matrices by solid-phase microextraction-transmission mode coupled to a portable mass spectrometer via direct analysis in real time. Analyst, 142(16), 2928-2935. https://doi.org/10.1039/C7AN00718C

González-Curbelo, M. Á., Socas-Rodríguez, B., Herrera-Herrera, A. V., González-Sálamo, J., Hernández-Borges, J., \& Rodríguez-Delgado, M. Á. (2015). Evolution and applications of the QuEChERS method. TrAC Trends in Analytical Chemistry, 71, 169_ 185. https://doi.org/10.1016/J.TRAC.2015.04.012

Goodley, S. (2017a). 2 Sisters boss apologises for 'mistakes' but dodges questions about breaches. The Guardian. Retrieved from https://www.theguardian.com/business/2017/oct/25/food-standards-bodies-culpable-in2-sisters-scandal-inquiry-hears

Goodley, S. (2017b). UK's top supplier of supermarket chicken fiddles food safety dates | Business | The Guardian. The Guardian. Retrieved from https://www.theguardian.com/business/2017/sep/28/uks-top-supplier-of-supermarketchicken-fiddles-food-safety-dates

Gredell, D. A., Schroeder, A. R., Belk, K. E., Broeckling, C. D., Heuberger, A. L., Kim, S.-Y., 

... Prenni, J. E. (2019). Comparison of Machine Learning Algorithms for Predictive Modeling of Beef Attributes Using Rapid Evaporative Ionization Mass Spectrometry (REIMS) Data. Scientific Reports, (1), 1. https://doi.org/10.1038/s41598-019-40927-6

Guitton, Y., Dervilly-Pinel, G., Jandova, R., Stead, S., Takats, Z., Le Bizec, B., \& Bizec, B. Le. (2017). Rapid evaporative ionisation mass spectrometry and chemometrics for highthroughput screening of growth promoters in meat producing animals. Food Additives \& Contaminants: Part A, 35(5), 1-11. https://doi.org/10.1080/19440049.2017.1421778

Huang, L.-F., Wu, M.-J., Zhong, K.-J., Sun, X.-J., Liang, Y.-Z., Dai, Y.-H., ... Guo, F.-Q. (2007). Fingerprint developing of coffee flavor by gas chromatography-mass spectrometry and combined chemometrics methods. Analytica Chimica Acta, 588(2), 216-223. https://doi.org/https://doi.org/10.1016/j.aca.2007.02.013

Kaufmann, A. (2012). The current role of high-resolution mass spectrometry in food analysis. Analytical and Bioanalytical Chemistry, 403(5), 1233-1249. https://doi.org/10.1007/s00216-011-5629-4

Lacina, O., Urbanova, J., Poustka, J., \& Hajslova, J. (2010). Identification/quantification of multiple pesticide residues in food plants by ultra-high-performance liquid chromatography-time-of-flight mass spectrometry. Journal of Chromatography A, 1217(5), 648-659. https://doi.org/https://doi.org/10.1016/j.chroma.2009.11.098

Lambropoulou, D. A., \& Albanis, T. A. (2007). Methods of sample preparation for determination of pesticide residues in food matrices by chromatography-mass spectrometry-based techniques: a review. Analytical and Bioanalytical Chemistry, 389(6), 1663-1683. https://doi.org/10.1007/s00216-007-1348-2

Lu, H., Zhang, H., Chingin, K., Xiong, J., Fang, X., \& Chen, H. (2018). Ambient mass spectrometry for food science and industry. TrAC Trends in Analytical Chemistry, 107, 99-115. https://doi.org/https://doi.org/10.1016/j.trac.2018.07.017 
Møller, J. K. S., Catharino, R. R., \& Eberlin, M. N. (2005). Electrospray ionization mass spectrometry fingerprinting of whisky: immediate proof of origin and authenticity. Analyst, 130(6), 890-897. https://doi.org/10.1039/B415422C

Nei, D., Nakamura, N., Ishihara, K., Kimura, M., \& Satomi, M. A rapid screening of histamine concentration in fish fillet by direct analysis in real time mass spectrometry (DART-MS). , 75 Food Control § (2017).

Raji, M. A., \& Schug, K. A. (2009). Chemometric study of the influence of instrumental parameters on ESI-MS analyte response using full factorial design. International Journal of Mass Spectrometry, 279(2), 100-106. https://doi.org/https://doi.org/10.1016/j.jms.2008.10.013

Riedl, J., Esslinger, S., \& Fauhl-Hassek, C. (2015). Review of validation and reporting of non-targeted fingerprinting approaches for food authentication. Analytica Chimica Acta, 885, 17-32. https://doi.org/https://doi.org/10.1016/j.aca.2015.06.003

Vaclavik, L., Rosmus, J., Popping, B., \& Hajslova, J. (2010). Rapid determination of melamine and cyanuric acid in milk powder using direct analysis in real time-time-offlight mass spectrometry. Journal of Chromatography A, 1217(25), 4204-4211. https://doi.org/10.1016/J.CHROMA.2010.03.014

Verplanken, K., Stead, S., Jandova, R., Poucke, C. Van, Claereboudt, J., Bussche, J. Vanden, ... Vanhaecke, L. (2017). Rapid evaporative ionization mass spectrometry for high-throughput screening in food analysis: The case of boar taint. Talanta, 169, 30-36. https://doi.org/10.1016/j.talanta.2017.03.056

Wei, S., Yao, W., Ji, W., Wei, J., \& Peng, S. (2013). Qualitative and quantitative analysis of anthraquinones in rhubarbs by high performance liquid chromatography with diode array detector and mass spectrometry. Food Chemistry, 141(3), 1710-1715. https://doi.org/https://doi.org/10.1016/j.foodchem.2013.04.074 
513 Yang, Y., Deng, J., \& Yao, Z.-P. (2015). Field-induced wooden-tip electrospray ionization 514 mass spectrometry for high-throughput analysis of herbal medicines. Analytica Chimica $515 \quad$ Acta, 887, 127-137. https://doi.org/https://doi.org/10.1016/j.aca.2015.06.025

516 Yong, W., Guo, T., Fang, P., Liu, J., Dong, Y., \& Zhang, F. (2017). Direct determination of 517 multi-pesticides in wine by ambient mass spectrometry. International Journal of Mass 518 Spectrometry, Vol. 417, pp. 53-57. https://doi.org///doi.org/10.1016/j.ijms.2017.03.005 